\title{
MEASUREMENT OF BODY TEMPERATURE OF PATIENTS IN A TERTIARY CARE HOSPITAL — AN OBSERVATIONAL STUDY
}

\author{
RUKHSANA PARVIN ${ }^{1}$, IRIN PERVEEN ${ }^{2}$, GOPAL BISWAS ${ }^{3}$, S.M. MAHBUBUR RAHMAN ${ }^{4}$, A.K.M. RAFIQUE UDDIN ${ }^{5}$
}

\begin{abstract}
Measurement of body temperature of a patient is a piece of clinical information. It can guide diagnostic and therapeutic measures by determining the presence of illness and the extent to which the patient is responding to treatment. So it should be measured accurately to identify fluctuations fast and intervene early. In the hospitals, temperature is recorded commonly by the nursing staff. The aim of this study was to observe the measurement technique of temperature by health personnel in different wards of a tertiary care hospital in Bangladesh. This prospective observational study was done among 50 nurses in Enam Medical College Hospital. A 9-point selfadministered questionnaire was supplied to the 3 working doctors in the departments of Medicine, Surgery and Obstetrics \& Gynecology. The duration of study was 60 days. The study revealed that $98 \%$ of the health personnel used mercury thermometer whereas $2 \%$ used digital thermometer, $96 \%$ selected axilla and $4 \%$ selected oral cavity for measuring temperature, $48 \%$ washed the device before using, 22\% explained the procedure to the patients or their attendants, only $6 \%$ asked if the patient had taken any cold or hot drinks within last 30 minutes or had done any exercise, $10 \%$ kept the thermometer at the site for 30 seconds, $76 \%$ for 1 minute, $2 \%$ for 1 minute and 30 seconds and 12\% for 2 minutes, 44\% washed the thermometer after using, 70\% nurses informed the patients of the temperature measurement, $88 \%$ documented the temperature value immediately after measuring. The study shows that current performance of our health personnel is very poor. Less manpower and overburdening with lot of patients might be contributing factors. Proper education and training can improve the overall situation and enable good practice.
\end{abstract}

\section{Introduction}

The presence of fever and its pattern and magnitude can give valuable clue to understand the nature and severity of illness. ${ }^{1}$ For complete ward round it is essential to scan the temperature chart routinely. Temperature measurement must be accurate and consistent as therapeutic interventions are based upon it. ${ }^{2}$ The site and measuring device chosen depend upon a number of factors including age, clinical condition, degree of accuracy required, safety and ease of use. It is a responsibility of the health care personnel to determine the best method to monitor temperature and to use the temperature monitoring device accurately. In the hospital wards,

1. Associate Professor, Department of Medicine, Enam Medical College \& Hospital, Savar, Dhaka

2. Associate Professor, Department of Gastroenterology, Enam Medical College \& Hospital, Savar, Dhaka

3. Indoor Medical Officer, Department of Medicine, Enam Medical College \& Hospital, Savar, Dhaka

4. Assistant Registrar, Department of Medicine, Enam Medical College \& Hospital, Savar, Dhaka

5. Professor, Department of Medicine, Enam Medical College \& Hospital, Savar, Dhaka temperature is measured usually by the nursing staff. The aim of this study was to observe the measurement technique of temperature by the nurses in different wards in Enam Medical College and Hospital.

\section{Materials and Methods}

This was a single blinded prospective observational study done among 50 nurses in Enam Medical College Hospital. The duration of study was 60 days. A 9-point self-administered questionnaire, pretested in volunteers for clear understanding and verified by a noninvestigator, was supplied to three working doctors in the department of Medicine, Surgery and Obstetrics \& Gynecology. The doctors were aware of the purpose of the study and filled in the questionnaire. The nurses did not know it. Temperature was measured by using mercury and digital thermometer. The results were recorded as percentage of the health personnel performing the duty data analyzing was done manually.

\section{Results}

During the study period there were 50 nurses in the concerned departments. Ninety eight percent of them used mercury thermometer whereas $2 \%$ used digital thermometer. Ninety six percent selected 
axilla and $4 \%$ selected oral cavity for measuring temperature. Forty eight percent washed the device before using and 22\% explained the procedure to the patients or their attendants. Only $6 \%$ asked if the patient had taken any cold or hot drinks within last 30 minutes or had done any exercise. Forty four percent washed the thermometer after using. Seventy percent informed the patient of the temperature measurement and $88 \%$ documented the temperature value immediately after measuring. Table I shows dwell time of thermometer in different sites of body.

Table I

Dwell time of thermometer in the examining sites of body

\begin{tabular}{lc}
\hline Time & Percentage \\
\hline 30 seconds & $10 \%$ \\
1 minute & $76 \%$ \\
1 minute 30 seconds & $02 \%$ \\
2 minutes & $12 \%$ \\
\hline
\end{tabular}

\section{Discussion}

Accurate temperature measurement is required in certain clinical situations or patients group. False high readings may lead to expensive and painful diagnostic studies and medical interventions. False low readings may lead to greater morbidity and mortality. In our study, $98 \%$ health personnel used mercury thermometer, though it is banned in many countries in the developed world due to environmental hazard done by the mercury. ${ }^{3}$ If the mercury glass thermometer breaks, it would cause spillage of the mercury which poses risk to public health. Two thermometers suitable for both hospital and community use are the battery operated electronic thermometer and the single use chemical thermometer. ${ }^{3}$ These are easy to use though battery also contains mercury and would be toxic for health if ingested specially in case of children. These thermometers are not available in all the pharmacies and are costlier than mercury thermometer.

Site of the body is an important determinant for measuring temperature. Though axilla is the most preferred site in hospital, it is a poor predictor of fever. ${ }^{4,5}$ Temperature cannot be measured accurately in axilla. ${ }^{6,7} \mathrm{~A}$ systematic review of 20 studies by Craig et al concluded that axillary thermometry is not a good method when accurate temperature measurements are required. ${ }^{8}$ Mercury glass thermometers can break if it is introduced in the rectum and there is also risk of cross infection. ${ }^{9}$ But rectal temperature can give accurate estimation, specially in neonates and young children. However, rectal measurement is debated due to risk of cross infection ${ }^{10}$ and it may be unacceptable and uncomfortable for older children and adults. ${ }^{11}$

Different sites require different times. The sensitivity differs according to site and duration. ${ }^{12}$ One must be aware of the recommended placement times for each type of thermometer, as keeping the thermometer in place for not long enough or for too long can affect the temperature reading adversely. ${ }^{13}$ To get the accurate measurement, thermometer should be kept in the mouth for about 10 minutes which is not practical. The time recommended in standard nursing texts varies between 2 and 7 minutes. ${ }^{3}$ The axilla being the preferred site for both aesthetic and safety reasons at the expense of accuracy and it requires 5-7 minutes for accurate measurement. In some studies the suggested time is 8 minutes. ${ }^{14,15}$ Rectal placement time should be 3 minutes, but may be more difficult to maintain. In our study, health personnel did not keep thermometers at the measuring sites for recommended period. So accuracy of the measurement remains doubtful.

Risk of nosocomial infection is high if proper hygiene is not maintained. Any devices should be disinfected before using in patients. Isopropyl alcohol is used for disinfection of clinical thermometer. Soaking in this solution for 10-15 minutes is sufficient for disinfection. ${ }^{16}$ Our study revealed that only 48 percent of the staffs disinfected device before using. Therefore, it poses great risk of infection. Thermometers should be washed after using, but only $44 \%$ did this procedure.

Before taking the temperature value, health personnel must explain the patients about the procedure. ${ }^{17}$ Our study shows only 22 percent of them talked to the patients regarding the procedure. Patients should be enquired about intake of any hot or cold drinks or foods before taking temperature orally as this may influence the value of measurement. ${ }^{16}$ Only $6 \%$ nurses asked patients regarding intake of hot or cold drinks or foods. Thus the accuracy of measuring temperature is questionable.

After taking temperature, nursing staff should inform the value of measurement to the patients. In our study $70 \%$ staff told the patients about their temperature value. Clinical decision is influenced by proper documentation of temperature. ${ }^{16}$ Our study revealed that $88 \%$ documented the temperature and rest of the staff did not keep any records.

Body temperature is one of the important vital signs which should be measured systematically in clinical 
practice. ${ }^{17}$ It has a great impact on medical care of the patients. Accuracy of this measurement is very essential. With the development of new measuring devices and appropriate technique, proper education and training are necessary for health personnel to evaluate temperature correctly.

\section{References}

1. Clarke S. Use of thermometers in general practice. BMJ 1992; 304(6832): 961-963.

2. Knies RC. Temperature measurement in acute Care: The who, what, where, when, why, and how? Available at: http://ENW.org/Research-Thermometry.htm. Accessed December 2012.

3. Blumenthal I. Should we ban the mercury thermometer? Discussion paper. Journal of the Royal Society of Medicine 1992; 85: 553-555.

4. Morley CJ, Thornton AJ, Cole TJ, Fowler MA, Hewson $\mathrm{PH}$. Axillary and rectal temperature measurements in infants. Arch Dis Child 1992; 67: 122-125.

5. Kresch MJ. Axillary temperature as a screening test for fever in children. I Pediazr 1984; 104: 596-599

6. Keeley D. Taking infants' temperatures. BMJ 1992; 304: 931-932.

7. Erickson RS, Woo TM. Accuracy of infrared ear thermometry and traditional temperature methods in young children. Heart Lung 1994; 23: 181-195.

8. Craig JV, Lancaster GA, Williamson PR, Smyth RL. Review temperature measured at the axilla compared with rectum in children and young people: systematic review. BMJ 2000; 320(7243): 1174-1178.
9. Krapp K (ed). Thermometer. In: Encyclopedia of Nursing \& Allied Health. Vol. 5. Gale Cengage, 2002. Available at: http://www.enotes.com/thermometer-reference.

10. Pazart L, Devilliers E, Bouté C, Aho S, Rupin C, Gouyon JB. What alternatives to rectal temperature recording with a mercury thermometer? Rev Epidemiol Sante Publique. 1997; 45(6): 516-526.

11. Prerna B, Abhijeet S, Moonis MAF. Thermometry in children. J Emerg Trauma Shock. 2012; 5(3): 246-249.

12. Textbook of Microbiology and Immunology- page xliiGoogle books. Available at: http://books.google.com/ books? Accessed December 2012.

13. Nursing Procedure: measuring and monitoring temperature in the highly dependent of critically ill infant or child - axilla temperature paediatric intensive care guideline. Available at: www.clinicalguidelines.scot.nhs.uk/.../YOR-PICU. Accessed November 2012.

14. Khorshid L, E er I, Zaybak A, Yapucu U. Comparing mercury-in-glass, tympanic and disposable thermometers in measuring body temperature in healthy young people. J Clin Nurs 2005; 14(4): 496-500.

15. Valter G, Toffanello E D, Enzi G, Perissinotto E, Vangelista S, Simonato M. Reliability of body temperature measurements in hospitalised older patients. Journal of Clinical Nursing 2008; 17(11): 1518-1525.

16. Mooney GP.Temperature 2007. Available at: www.nursingtimes.net/temperature/200193. Accessed December 2012.

17. McCallum L, Higgins D. Measuring body temperature. Nurs Times 2012; 108(45): 20-22. 